
MACIEJ GRABOWSKI

\title{
Modern methods of treatment of post-traumatic bone defects in Department of Trauma Surgery and Emergency Medicine, Medical University of Lublin
}

\begin{abstract}
Motor organ injuries are one of the most common consequences of trauma, out of which comminuted fractures with a bone loss are the most challenging. Numerous methods are applied to treat these injuries; however, still in many cases we are unable to suggest successful medical treatment. Therefore, treating these injuries using elastic and surgically handleable bone replacement materials was started at Trauma Surgery and Emergency Medicine of Medical University of Lublin (provided for the experiments by Medical Inventi Lt). Preliminary assessment based on the results of treatment with bone replacement materials of two patients with comminuted fractures of femur was promising. Bone union was achieved without any adverse effects.
\end{abstract}

Keywords: fracture, bone substitutes, bone grafts.

DOI: $10.1515 /$ pjph-2015-0022

\section{INTRODUCTION}

Injuries to the musculoskeletal system are one of the most common causes of disability. It is estimated that some four million people suffer from temporary or permanent disabilities related to musculoskeletal injuries every year. Failures of treatment for fractures of long bones (e.g. delayed union, pseudoarthrosis or osteomyelitis,) are one of the leading causes of musculoskeletal disorders. Frequently, these injuries are associated with bone loss, out of which comminuted fractures with a severe bone loss are the most challenging [1].

The treatment of these fractures is a multi-stage process lasting for months or even years and the final result is uncertain. Methods that are commonly used to treat such fractures allow for positive outcome. Using bone replacement materials sheds a promising light on achieving bone union, especially that not too long ago, amputation was the only solution in such cases Hence, this treatment method might have some influence on the development of trauma and reconstructive surgery [2].

Reparative ability of the skeletal system is of particular importance to modern orthopedics. Calcium phosphatebased bone substitutes, namely hydroxyapatite bioceramics, should be distinguished from other bone substitute materials due to their biocompatibility with natural bone apatite, which constitutes a non-biological component of bone and teeth $[2,3]$. These materials have been used in dentistry since the 1980s and they show great bioactivity It is assumed that bone replacement materials can fully integrate with the bones and no connective-tissue sheath is needed.
Moreover, these materials have proven to be non-irritant, without any cytotoxic or cancerogenic effects. Hydroxyapatite bioceramics arechemically pure and do not transform during sterilization. When introduced into bone defect it reduces the bleeding and the amount of thrombus, therefore they decrease septic complications and promote tissue regeneration. Furthermore they decrease osteoclast activity in favor of osteoblast induction $[4,5]$.

In future clinical studies concerning bone regeneration after surgical procedures, a composite material developed and patented in the Department of Biochemistry and Biotechnology, Faculty of Pharmacy and Division of Laboratory Medicine, Medical University of Lublin will be used. This is a two-phase micro- and macroporous composite with some properties similar to human bones. Its production was based on hydroxyapatite granulate and non-toxic organic carbohydrate polymer. Hydroxyapatite which constitutes a major element of biomaterial is characterized by high biocompatibility, non-toxicity and lack of immunogenicity $[4,5]$.

Due to its composition the material does not reveal disadvantages attributed to hydroxyapatite bioceramics, as an excessive rigidity and lack of surgical handiness. Moreover it has low plasticity, is susceptible to formation and easily adapts to the size of the defect $[4,5]$.

Mechanical parameters of biomaterial resemble those of cancellous bone, it is non-toxic to human osteoblasts (demonstrated in vitro) and constant in the process of sterilization. It can also serve as a carrier for drugs (antibiotics, protein growth factors) contributing to antimicrobial protection of implantation site and stimulating the healing process. When introduced into the bone defect it will serve as a scaffold or platform for osteoblasts and thus it will provide osteoconductivity $[4,5]$. 


\section{AIM}

The aim of this study is to clinically evaluate the bonereplacement two-phase composite material as a filler in posttraumatic primary and secondary bone defects, as a modern method used in traumatology and orthopedics.

\section{MATERIAL AND METHODS}

Patients were selected for the study, based on the presence of primary and secondary bone defects. Two patients were enrolled into the preliminary phase of the study. Written consent was collected from all patients. Elastic hydroxyapatitepolymeric biocomposite (provided by Medical Inventi Lt) was used as an implantable material.

Bone-replacement material was administrated during scheduled surgical procedures under general anesthesia. The composite was introduced in primary or secondary bone defect at the end of the surgery. The amount of agent used was dependent on shape and size of bone defect. Each patient underwent clinical examination during the $1^{\text {st }}, 3^{\text {rd }}, 6^{\text {th }}$ and $12^{\text {th }}$ month post-surgery. Potential adverse effects of the treatment were taken into consideration while evaluating its outcome.

Physical examination included clinical examination of the limb, where the composite was introduced (swelling, inflammatory response and tenderness were evaluated). Furthermore, the limb function was estimated. Conventional X-ray and CT scans ware collected in order to assess bone union and bone remodeling. Moreover, serum levels of calcium, alkaline phosphatase and C-reactive protein was evaluated on every clinical examination.

\section{RESULTS}

Twenty-five patients will be selected for the final study group. In this study we present preliminary results of first two patients who were qualified and treated with the study regimen. All surgical procedures were performed in Trauma Surgery Clinic of Medical University of Lublin in 2013. In these surgeries, bone-replacement material, (developed in Biochemistry Department of Medical University of Lublin and supplied by Medical Inventi Lt.) was applied. Both patients suffered from posttraumatic bone loss and bone union disorders. The size of the bone defect determined the shape and size of implant used. The sizes varied from 1 to $10 \mathrm{~cm}$, with average $1 \mathrm{~cm}$.

The first patient was a 61-year-old male who suffered from an extensive injury of his left femur as a result of traffic accident (multifragmentary fracture of left femur) in 2012. Initially, the patient was treated surgically by open reduction with internal fixation with LCP plate and screws. After a few months bone union was not achieved on the medial aspect of distal metaphysis along with intense pain. The effect of previous treatment was unsatisfying. The patient underwent a successive surgery in April 2013 in Trauma Surgery and Emergency Medicine Clinic. The bone defect site was exposed and all dead bone and necrotic tissue was removed. In the next step the bone substitute was introduced into the defect site. Prior to introduction the composite was immersed in patient's blood, accurately cut and trimmed to fit the bone defect. A cylinder-shaped block with a diameter of $1 \mathrm{~cm}$ and length of $10 \mathrm{~cm}$ was used. Afterwards the defect site was covered with muscles, fascia, subcutaneous tissue and skin.

The second patient was a 25-years-old male, who suffered from multiple fractures in a traffic accident in 2010. After preliminary fixation in the district hospital the patient was transported to the Clinic. Upon the admission, intertrochanteric fracture of right femur, fracture of right tibial plateau, fracture of right ulna and radius shafts and multifragmentary open fracture of distal 1/3 of right femur diaphysis was diagnosed.

The patient underwent a surgery - open reduction with internal fixation of femur and tibia fractures with LCP plates. Fractures of forearm were also stabilized. Bone union was achieved in all fractures apart from the distal third of femur diaphysis. No matter what techniques would have been applied, it would never be possible to obtain bone union The patient selected for the study underwent a surgery in May 2013, during which, bone replacement materials were used. The defect site was exposed. All dead bone and adjacent necrotic tissue was removed. After that, a block sized $8 \times 1 \mathrm{~cm}$ was introduced into the bone.

The results of both patients' treatment were radiologically assessed during the $1^{\text {st }}, 3^{\text {rd }}, 6^{\text {th }}$ and $12^{\text {th }}$ month following the surgery. Moreover, biochemical analysis of serum levels of alkaline phosphatase (ALP), ALP liver and bone fraction, phosphate, calcium and C- reactive protein was evaluated during similar intervals. The biochemical analysis showed no abnormalities in either patient.

Successive X-rays showed progressive bone union and that bone replacement material was incorporated into the bone. Even though bone union and ability do full weight bearing was achieved, the second patient was re-operated due to malunion and wrong implant placement. The LCP plate was removed and other fixation device was used.

There was neither an inflammatory response to the material in patients nor were there any adverse effects. The course of the surgery was proper and as planed preoperatively on X-rays, CT scans and physical examination. The material used in the study is easy to mold, which means that it is suitable for filling bone defects of various shapes and sizes. There is a huge necessity for reconstructive bone procedures and while preliminary results of the treatment are encouraging, it seems that such procedures might be widely implemented in the future. Apart from radiological assessment patient underwent biochemical evaluation which has shown no significant problems.

To conclude, the preliminary results of treatment implementing the bone-replacement material are encouraging and the use of the composite seems to be a safe and easy-touse solution.

\section{DISCUSSION}

Given that comminuted fractures of long bones often result from a high-energy trauma, and therefore are accompanied by bone losses, delayed union and pseudoarthrosis, it is of huge importance in trauma, orthopaedic and reconstructive surgery to fill the bone defects and achieve a bone un- 
ion which promotes favorable functional effect of treatment. In many patients autological bone grafts harvested from iliac crest or tibial tuberosity are utilized [6]. However, in such cases bone tissue limitations can occur, which means that only minor defects could be healed using the technique. Moreover, many patients underwent previous surgeries in which autogeneic bone grafts ware already harvested, and this technique is not suitable for use.

Allogeneic frozen bone transplants could provide an alternative. Frozen bones are devoid of vivid cells which means that they can be safely used in fractures treatment. In addition, a frozen bone has a range of supportive properties. When a frozen bone graft is introduced into the bone defect site, it provides additional mechanical stability. Furthermore, frozen bone grafts have osteoconductive properties, due to which osteoblasts infiltrate the surface of implant, and osteoblasts proliferate in damaged bone tissue. Despite all the advantages that frozen bone transplants have, the costs and difficulty in molding the graft are limiting the use of this technique.

In our opinion, modern medicine is still lacking an efficient and available method of treatment of posttraumatic bone defects. We believe that a bone-replacement material based on hydroxyapatite granulate and non-toxic carbohydrate organic polymer, which was used in this study, might be a solution in difficult cases. This is an original implantable material of 3rd generation due to its surgical handiness and bone tissue regeneration capacity. The ease of molding, cutting and filling irregular defects gives the surgeon comfort while performing a surgery. Moreover, no adverse effects such as inflammatory response or infection were noted.

According to earlier findings [4,5], the composite, due to high hydroxyapatite content, is highly biocompatible, nontoxic and is lacking of immunogenicity. When implemented the material will act as a scaffold or a bridge for osteoblasts, therefore it will act in osteoconductive way which can significantly support the process of bone reconstruction $[4,5]$. Additionally it is possible to soak the material in any kind of antibiotic used in treating osteomyelitis which commonly accompanies comminuted fractures with a severe bone loss. It seems that poor mechanical properties of this composite are its greatest disadvantage. Moreover, the material becomes even softer when immersed in blood, therefore it is necessary to use the material with fixation devices which provide stability of the fracture. Nonetheless, it is possible to combine the composite with a titanium core, which would provide adequate stability. However, at this moment it is only a theoretical possibility for future development.

All patients were evaluated clinically, biochemically and radiologically prior to surgery and post surgery in strict intervals. There were no anomalies found, which means that we can consider using bone-replacement material as safe for patients.

\section{CONCLUSIONS}

Preliminary assesment based on the results of treatment of the post-traumatic bone defences with bone-replacement materials are promising. A wider application, however, of this modern method in traumatology and orthopedics requires further research.

\section{REFERENCES}

1. Marczyński W. Współczesne poglądy patogenetyczne a biologia zaburzeń zrostu kości długich. Ortop Traumatol. 2006;3:7-14.

2. Axelrad T, Kakar S, Einhorn T. New technologies for the enhancement of skeletal repair. Injury Int J Care Injured. 2007;38(Suppl.1):49-62.

3. Hamera-Słynarska M. Farmakologiczne wspomaganie gojenia złamań. Ortop Traumatol Rehab. 2000;3:11-4.

4. Belcarz A, Ginalska G, Pycka T, et al. Application of $\beta$-1,3-glucan in production of ceramics-based elastic composite for bone repair. Centr Eur J Biol. 2013;8:534-48.

5. Belcarz A, Zima A, Ginalska G. Biphasic mode of antibacterial action of aminoglycoside antibiotics-loaded elastic hydroxyapatite-glucan composite. Intern J Pharm. 2013;454:285-95.

6. Sen MK, Micalu T. Autologous iliac crest bone graft: should is still be the gold standard for treating nonunions? Injury Int J Care Injured. 2007;38(Suppl. 1):75-80.

\section{Corresponding author}

Mariusz Jojczuk

Department of Trauma Surgery and Emergency Medicine,

Medical University of Lublin

16 Staszica Str.,20-081 Lublin

tel +48 081 53-218-54

E-mail: mariuszjojczuk@o2.pl 